\title{
Chromosome-autonomous feedback down- regulates meiotic DNA break competence upon synaptonemal complex formation
}

\author{
Xiaojing Mu, ${ }^{1,2}$ Hajime Murakami, ${ }^{2}$ Neeman Mohibullah, ${ }^{2,3,4}$ and Scott Keeney ${ }^{1,2,3}$ \\ ${ }^{1}$ Weill Cornell Graduate School of Medical Sciences, Cornell University, New York, New York 10021, USA; ${ }^{2}$ Molecular Biology \\ Program, Memorial Sloan Kettering Cancer Center, New York, New York 10065, USA; ${ }^{3}$ Howard Hughes Medical Institute, \\ Memorial Sloan Kettering Cancer Center, New York, New York 10065, USA
}

\begin{abstract}
The number of DNA double-strand breaks (DSBs) initiating meiotic recombination is elevated in Saccharomyces cerevisiae mutants that are globally defective in forming crossovers and synaptonemal complex (SC), a protein scaffold juxtaposing homologous chromosomes. These mutants thus appear to lack a negative feedback loop that inhibits DSB formation when homologs engage one another. This feedback is predicted to be chromosome autonomous, but this has not been tested. Moreover, what chromosomal process is recognized as "homolog engagement" remains unclear. To address these questions, we evaluated effects of homolog engagement defects restricted to small portions of the genome using karyotypically abnormal yeast strains with a homeologous chromosome $\mathrm{V}$ pair, monosomic V, or trisomy XV. We found that homolog engagement-defective chromosomes incurred more DSBs, concomitant with prolonged retention of the DSB-promoting protein Rec114, while the rest of the genome remained unaffected. SC-deficient, crossover-proficient mutants ecm11 and gmc2 experienced increased DSB numbers diagnostic of homolog engagement defects. These findings support the hypothesis that SC formation provokes DSB protein dissociation, leading in turn to loss of a DSB competent state. Our findings show that DSB number is regulated in a chromosome-autonomous fashion and provide insight into how homeostatic DSB controls respond to aneuploidy during meiosis.
\end{abstract}

[Keywords: aneuploidy; double-strand breaks; meiosis; recombination; Spo11; synaptonemal complex; trisomy]

Supplemental material is available for this article.

Received July 27, 2020; revised version accepted September 29, 2020.

Programmed DSB formation by Spo11 initiates meiotic homologous recombination (Keeney et al. 2014). A fraction of DSBs are repaired as crossovers, contributing to physical linkages essential for proper segregation of homologs in meiosis I. Although DSBs can be deleterious, meiotic cells make them in large numbers (150-200 DSBs per cell in S. cerevisiae) (Buhler et al. 2007; Pan et al. 2011). To mitigate the risk of genome instability, DSB formation is controlled quantitatively, spatially, and temporally by a network of intersecting feedback circuits (Keeney et al. 2014).

One circuit involves inhibition of DSB formation after homologous chromosomes have engaged one another. In S. cerevisiae, mutants lacking any of several ZMM proteins exhibit elevated DSB levels (Thacker et al. 2014). ZMM proteins (Zip1-4, Msh4-5, Mer3, Spo16, and

\footnotetext{
${ }^{4}$ Present address: Integrated Genomics Operation, Memorial Sloan Kettering Cancer Center, New York, NY 10065, USA.

Corresponding authors: s-keeney@ski.mskcc.org, murakamh@mskcc.org Article published online ahead of print. Article and publication date are online at http://www.genesdev.org/cgi/doi/10.1101/gad.342873.120. Freely available online through the Genes \& Development Open Access option.
}

Pph3) promote formation of type I (interfering) crossovers and assembly of $\mathrm{SC}$, a proteinaceous structure that includes the aligned axes of homologs plus the central region components that hold them together (Pyatnitskaya et al. 2019). The elevated DSBs in ZMM mutants suggest that DSB control is defective when either synapsis or crossing over (or both) is impaired. A similar conclusion was reached on the basis of additional DSB formation in mutants with recombination defects from altered usage of strand exchange proteins (Lao et al. 2013). In mouse spermatocytes, chromosome segments that fail to synapse-either naturally on the nonhomologous parts of the $\mathrm{X}-\mathrm{Y}$ pair or in response to a recombination-defective mutation-accumulate a higher density of foci of the strand exchange protein RAD51, a cytological marker for DSBs (Kauppi et al. 2013). A prolonged period of DSB formation has also been proposed to account for elevated DSB markers in Caenorhabditis elegans mutants with synapsis and/or crossover defects (e.g., Henzel et al. 2011).

(C) $2020 \mathrm{Mu}$ et al. This article, published in Genes \& Development, is available under a Creative Commons License (Attribution 4.0 International), as described at http://creativecommons.org/licenses/by/4.0/. 
To explain the yeast findings, we proposed that a ZMMdependent process feeds back to inhibit DSB formation (Keeney et al. 2014; Thacker et al. 2014). The molecular identity of this process was (and remains) undefined, so we used the mechanistically ambivalent term "homolog engagement" to describe it. One possibility is SC formation, consistent with the behavior of asynaptic chromosome segments in mice (Kauppi et al. 2013). Indeed, earlier work showed that synapsis in mice is followed by displacement from chromosomes of the DSB-promoting axis protein HORMAD1 (Woitasz et al. 2009). This observation led to the proposal that SC formation down-regulates DSB formation by removing proteins needed for SPO11 activity (Wojtasz et al. 2009), an idea independently proposed later on the basis of unsynapsed regions in yeast retaining Spo11 accessory proteins such as Rec114 (Panizza et al. 2011; Carballo et al. 2013).

A nonexclusive alternative is that some aspect of crossover formation is the trigger for ZMM-dependent feedback (Keeney et al. 2014; Thacker et al. 2014). In C. elegans, prolonged chromosome binding of DSB-promoting proteins DSB-1 and DSB-2 occurs in mutants that cannot make crossover-designated recombination intermediates, even if SC is formed (Rosu et al. 2013; Stamper et al. 2013). This regulation involves the kinase CHK-2 and occurs nucleus-wide in response to a crossover defect on just a single chromosome pair (Carlton et al. 2006; Rosu et al. 2013; Stamper et al. 2013). In contrast, in mice, CHK2 does not appear to regulate DSB number (Pacheco et al. 2015) and the increase in DSB levels caused by asynapsis appears to be restricted to unsynapsed regions; i.e., is chromosome autonomous (Kauppi et al. 2013). Whether yeast DSB control more closely resembles C. elegans or mice (or neither) is not yet clear.

One limitation has been that most available data in yeast are from strains with catastrophic meiotic failure (e.g., ZMM mutants) that can simultaneously impinge on multiple feedback circuits including hyperactivation of Tel1 (ATM)- and Mec1 (ATR)-regulated pathways (Cooper et al. 2014; Keeney et al. 2014). To circumvent this limitation, we used here a set of karyotypically abnormal S. cerevisiae strains in which defects in synapsis and/or crossing over are confined to specific parts of the genome through sequence divergence or chromosome gain or loss. This experimental setting enabled us to demonstrate that feedback through homolog engagement is chromosome autonomous and is accompanied by removal of Rec114 from chromosome pairs that have engaged one another. Additionally, analysis of SC-deficient but crossover-proficient ecm $11 \Delta$ and gmc2 $\Delta$ mutants showed that SC formation is essential and that crossing over without SC formation is not sufficient to support feedback control of DSB numbers.

\section{Results}

Karyotypically abnormal S. cerevisiae strains

The karyotype abnormalities we studied and their predicted effects on homolog engagement are cartooned in Figure
A
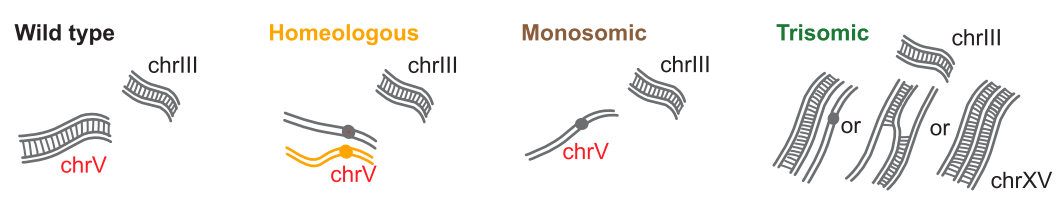

B
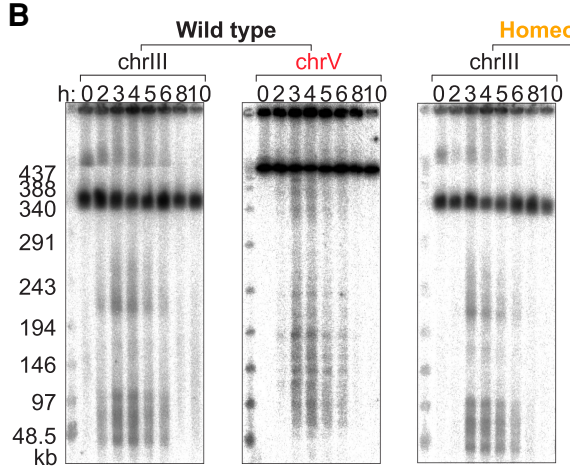

C
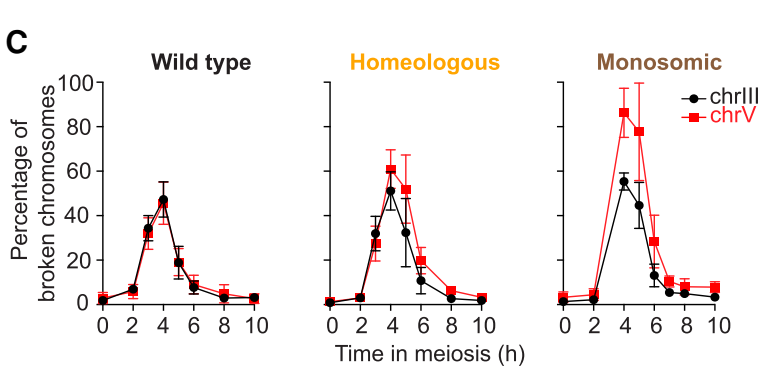

Figure 1. Higher DSB levels on homolog engagement-defective chromosomes. (A) Cartoons of wild-type, homeologous, monosomic, and trisomic chromosome configurations. Gray lines are S. cerevisiae chromosomes and orange lines are S. pastorianus. The homeologous chrV pair rarely synapses or recombines. The trisomic chromosomes can adopt different synaptic configurations ("II $+\mathrm{I}$," partner switch, and triple synapsis). (B) Representative PFGE Southern blots probed for chrIII and chrV. P, signal from parental-length $\mathrm{DNA}$; $\mathrm{W}$, signal in wells. Asterisk indicates an ectopic recombination product between leu2::hisG (on chrIII) and ho::his $G$ (on chrIV) in the monosomic strain. The other strains do not form this product because they do not have a his $G$ insert at ho. (C) Poisson-corrected DSB quantification of PFGE Southern blots. (D) Quantification of time-averaged DSBs on chrV relative to chrIII for wild-type, homeologous, and monosomic strains. $\left(^{*}\right) P<0.05$, unpaired $t$-test. Error bars in $C$ and $D$ are mean \pm SD except for the monosomic strain (mean \pm range). 
1A. Homolog engagement-proficient parts of the genome serve as internal controls to counter culture-to-culture variation in meiotic timing or efficiency, making these systems sensitive enough to detect even small differences.

The homeologous strain contains one copy of chromosome $\mathrm{V}(\mathrm{chrV})$ introgressed from $S$. pastorianus (also known as $S$. carlsbergensis) in an otherwise $S$. cerevisiae background (Dawson et al. 1986; Nilssontillgren et al. 1986; Goldman and Lichten 2000; Maxfield Boumil et al. 2003; Kemp et al. 2004). The homeologous chrV copies differ by $\sim 30 \%$ from one another (Maxfield Boumil et al. 2003). As a result, they show little if any evidence of homologous pairing or complete synapsis along their lengths and only very rarely produce crossovers despite making DSBs (Dawson et al. 1986; Nilssontillgren et al. 1986; Goldman and Lichten 2000; Maxfield Boumil et al. 2003; Kemp et al. 2004; Newnham et al. 2010).

The monosomic strain was generated by inducing loss of one copy of chrV before meiotic entry using a centromere-destabilizing system in which an inducible $G A L$ promoter drives transcription across the centromere (Hill and Bloom 1987). We developed a presporulation procedure that yields efficient chromosome loss (Supplemental Fig. S1; Supplemental Table S1; see the Materials and Methods).

Unlike zip3 mutants (Börner et al. 2004), the homeologous and monosomic strains progressed through meiosis without a strong arrest in prophase I (Supplemental Fig. S2A). However, both strains exhibited a moderate delay $(\sim 2 \mathrm{~h})$ in nuclear division, likely from the spindle assembly checkpoint responding to nonexchange chromosomes (Marston and Wassmann 2017). The DSBs on these chromosomes are presumably repaired by recombination between sister chromatids (de Massy et al. 1994; Hochwagen et al. 2005; Goldfarb and Lichten 2010).

We also present data from a serendipitously generated strain bearing three copies of chrXV. Trisomic chromosomes can form various synaptic configurations such as a fully synapsed pair plus a completely unsynapsed chromosome ("II + $\mathrm{I}^{\prime}$ ), synaptic partner switches, or triple synapsis along the entire chromosome length (Goldstein 1984; Loidl 1995). In "II + I" or synaptic partner switch configurations, at least one of the three homologs lacks a partner at every position along the chromosome length (Fig. 1A).

\section{More DSBs for longer times on homeologous and monosomic chromosomes}

If homolog engagement functions chromosome-autonomously in cis, the homeologous and monosomic chromosomes should experience prolonged and more DSB formation compared with engagement-proficient chromosomes. To test this, we measured DSBs across meiotic time courses by separating high molecular weight DNA using pulsed-field gel electrophoresis (PFGE) followed by Southern blotting and indirect end-labeling (Fig. 1B). For each culture, we quantified DSBs on chrV (wild type, homeologous, or monosomic) and on chrIII as an internal control (Fig. 1C ; Supplemental Fig. S2B-D). To standardize quantitative comparisons despite different chrV copy numbers, we probed for a nat $M X 4$ cassette integrated near the right end of one (or the only) S. cerevisiae copy of chrV. ChrIII was detected using a probe for CHA1 on the left arm. DSB frequencies (broken DNA molecules as percent of total DNA) were corrected for multiple breaks on the same chromatid using a Poisson approximation (Murakami and Keeney 2014).

In wildtype, chrIII and chrV coincidentally formed DSBs with indistinguishable amounts and timing (Fig. 1C; Supplemental Fig. S2B). In contrast, the homeologous and monosomic strains reproducibly displayed higher levels of broken chrV compared with chrIII. Similar increases were apparent $(\sim 1.2$-fold for homeologous, $\sim 1$.7-fold for monosomic) whether considering peak values or time-averaged DSBs (area under the curve) (Fig. 1C,D; Supplemental Fig. S2C,D). The greater effect for monosomic chrV than for homeologous may reflect a difference in degrees of homolog engagement defect, because the homeologous chromosome pair still has some level of sequence similarity, whereas the monosomic chromosome completely lacks a pairing partner.

We also noted that the homeologous strain had similar DSB amounts on chrV and chrIII at the earliest time points (2 and $3 \mathrm{~h}$ ), with chrV diverging from chrIII later (Fig. 1C; Supplemental Fig. S2C). Moreover, we observed a reproducible delay of $\sim 15$ min on homeologous chrV compared with wild type when estimating DSB peak signal times by curve fitting (Supplemental Fig. S2E,F). (Absence of 3-h time points precluded this analysis for the monosomic cultures.)

These results are consistent with the prediction that homeologous and monosomic chromosomes should accumulate more DSBs because of prolonged DSB formation. However, an alternative interpretation could be that DSB numbers are the same, but DSB lifespan has been increased because of a repair delay caused by absence of a homolog as a repair partner (de Massy et al. 1994; Hochwagen et al. 2005). We did not favor this alternative as the sole explanation because repair using the sister chromatid can be rapid and efficient if a homolog is not available (Goldfarb and Lichten 2010). Nevertheless, we addressed this question more directly using an orthogonal approach to quantifying relative DSB formation: Spo11-oligonucleotide (oligo) sequencing.

\section{A homeologous chromosome pair incurs more DSBs}

Spo11 cleaves DNA in a topoisomerase-like manner, creating a covalent bond at the $5^{\prime}$-strand termini of DSBs that is then clipped endonucleolytically to release Spo11 still attached to a short oligo (Supplemental Fig. S3A; Neale et al. 2005). Spo11-oligo complexes are a quantitative byproduct of DSB formation and their lifespan is not tied to that of DSBs (Thacker et al. 2014). We immunoprecipitated FLAG-tagged Spol1 from the homeologous strain harvested at 4 and $5 \mathrm{~h}$ in meiosis, then purified Spo11 oligos to prepare libraries for Illumina sequencing and compared with existing wild-type maps generated using a protein A-tagged version of Spo11 (Supplemental Fig. S3A; Thacker et al. 2014; Mohibullah and Keeney 2017). 
Spo11-oligo maps from the wild-type and homeologous strains agreed well on chrIII (an internal control), exhibiting peaks (hot spots) with similar distributions at kilobase size scales (Supplemental Fig. S3B).

To compare per-chromosome distributions of Spo11 oligos, we first normalized the total number of sequence reads from each data set to one million (reads per million, RPM). If a specific chromosome (namely, chrV) generated more Spo11-oligo complexes, RPM on the other chromosomes would decrease even if the actual number of Spo11 oligos generated in vivo was unchanged. To account for this, we further scaled each data set to have an equal number of total RPM coming from the 15 chromosomes other than chrV. The scaled RPM was then summed for each chromosome. For the homeologous strain, we summed reads for both $S$. pastorianus and $S$. cerevisiae chrV.

As expected, the 15 chromosomes other than chrV aligned well with the diagonal when wild-type and the homeologous strain were compared (gray points in Fig. 2A). This indicates that the relative number of Spo11-oligo reads between chromosomes is reproducible if they have a homologous partner. ChrV also fell on the diagonal at $4 \mathrm{~h}$, but it deviated substantially at $5 \mathrm{~h}$, with a 1.7 -fold higher number of Spol1 oligos than expected from behavior of the homologous pair in wild type (orange points in Fig. 2A,B).

Because we are measuring normalized rather than absolute Spo11-oligo frequencies, we cannot exclude the possibility that the homeologous strain experiences changes in absolute DSB numbers on the other 15 chromosomes. However, such a change would have had to affect all of the chromosomes in close proportion to their DSB levels in wild type to maintain the good overall fit between the data sets (Fig. 2A). Therefore, these results strongly indicate that a homeologous chromosome pair selectively generates higher numbers of DSBs in a chromosome-autonomous fashion. The time dependence further supports the conclusion that homolog engagement defects allow DSBs to continue forming after they would normally have stopped.

\section{Trisomy also triggers elevated DSB formation}

A further test of the homolog engagement model came from a strain containing a trisomic chrXV pair. In the course of other studies (Mohibullah and Keeney 2017), we observed that Spo11-oligo maps from a particular culture of a supposedly wild-type strain exhibited abnormally high read counts on chrXV. Other clones from the same stock behaved normally (Mohibullah and Keeney 2017), so we speculated that the exceptional culture might harbor a spontaneous aneuploidy for chrXV. Indeed, quantitative Southern blotting after PFGE revealed three copies of chrXV (Supplemental Fig. S3C). Attempts to obtain other aneuploid clones from the stock failed, so the trisomy-XV strain exists now only in its Spo11-oligo maps at 4, 5, and $6 \mathrm{~h}$. These maps proved informative about the effects of aneuploidy on homolog engagement.

To compare per-chromosome distributions, we applied a two-step normalization similar to the one described above: RPM normalization followed by scaling to set the total number of reads from the 15 chromosomes other than chrXV equal between wild-type and the trisomic strain. To correct for chromosome copy number, reads from trisomic chrXV were further scaled by a factor of $2 / 3$. Even after correction, chrXV reads were overrepresented by twofold at $5 \mathrm{~h}$ (Fig. 2C). Again, overrepresentation was time dependent in that Spol1-oligo counts were only $\sim 1.4$-fold higher at $4 \mathrm{~h}$ (Fig. 2B,C). Counts remained approximately twofold elevated at $6 \mathrm{~h}$; we interpret the lack of further increase from 5 to $6 \mathrm{~h}$ as a consequence of Ndt80-driven exit from prophase I and concomitant down-regulation of DSB formation. In other words, prophase I exit ends the window of
A

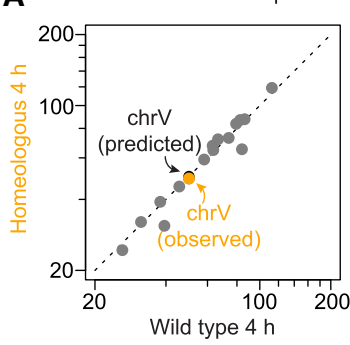

Spo11-oligo counts (kRPM)

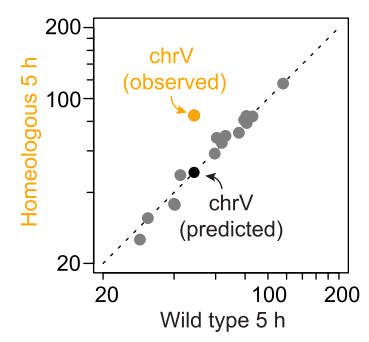

Spo11-oligo counts (kRPM)
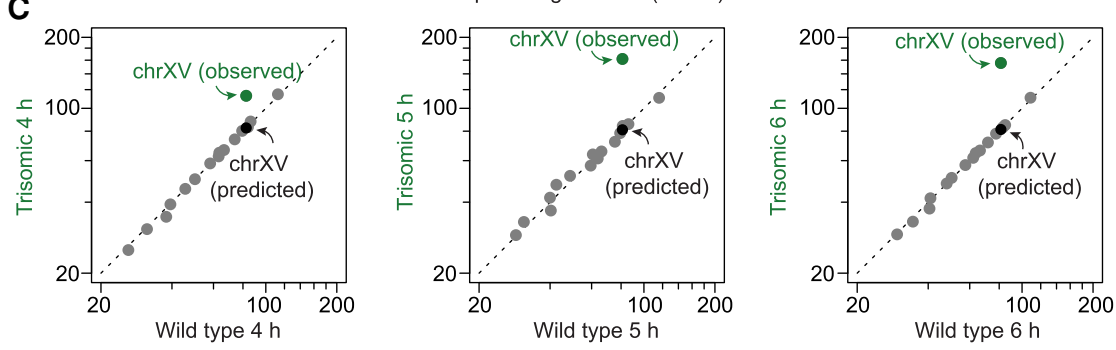

Figure 2. Increased DSBs are observed specifically on homeologous chrV and trisomic chrXV. $(A, C)$ Comparison of per-chromosome Spo11-oligo totals (kRPM [thousands of reads per million reads mapped]) between homeologous and wild type in $A$ ( $4 \mathrm{~h}[\mathrm{left}] ; 5 \mathrm{~h}[$ right $]$ ), and between trisomic and wild type in $C(4 \mathrm{~h}$ [left $] ; 5 \mathrm{~h}$ [middle]; $6 \mathrm{~h}[$ right $])$. Each dot represents one chromosome. A predicted data point assuming that the homeologous chrV or trisomic chrXV has the same number of reads as the homologous chrV or chrXV is shown in each graph. The deviation of two chromosomes (chrIX and chrXII) from the diagonal line in $A$, left, are likely due to a small difference in timing between the two cultures; these chromosomes are known to experience DSB formation relatively late on average (Murakami et al. 2020). (B) The fold difference between experimental and predicted values for the karyotypically abnormal strains (from $A$ and $C)$ at different meiotic times. Dashed line marks no change, meaning the experimental data matches prediction. 
opportunity during which homolog engagement defects can allow DSBs to accumulate.

\section{Increased DSB formation is not due to appearance of new hot spots}

The increase in Spol1 oligos on homeologous chrV or trisomic chrXV could reflect appearance of additional DSB hot spots or an increase of DSBs in the same hot spots. To differentiate between these possibilities, we called hot spots using an algorithm that identifies sites with Spo11-oligo counts above a threshold of 2.3 times the genome average (Pan et al. 2011). This identified 222 hot spots on $S$. cerevisiae chrV in the homeologous strain and 185 in wild type (Fig. 3A). However, the 37 hot spots called uniquely in the homeologous strain were weak ones that in wild type also had clusters of Spol1-oligo reads below the hot spot-calling threshold (Fig. 3B,C). Thus, the apparent increase in hot spot number on chrV when it is combined with a homeologous partner is a consequence of applying an arbitrary hot spot-calling threshold when there is a general increase in DSBs specifically on that chromosome. A similar conclusion was drawn for trisomic chrXV (Fig. 3D-F). We conclude that homolog engagement defects lead to increased DSB formation principally within existing hot spots rather than creating new sites of preferential Spo11 action.

\section{Homeology does not further increase DSB formation in a zip3 background}

If the increased DSB formation on homeologous chromosomes reflects loss of the same feedback loop that is defective in ZMM mutants, as we hypothesized, then a ZMM mutation should be epistatic with homeology. That is, the global homolog engagement defect in a ZMM mutant would mean that a homeologous chromosome pair would not behave differently from homologous pairs.

To test this prediction, we generated Spo11-oligo maps from a zip3s strain carrying the homeologous chrV pair. Zip3 is a SUMO E3 ligase required for SC polymerization, and deletion of ZIP3 leads to severe meiotic division delay, low spore viability, reduced crossing over, and incomplete SC formation (Agarwal and Roeder 2000; Perry et al. 2005; Cheng et al. 2006). The zip34 strain carrying the homeologous chrV pair showed meiotic arrest similar to zip3s (Supplemental Fig. S2A). Homologous and homeologous chrV pairs exhibited similar numbers of Spol1oligo reads in the zip3 $\Delta$ background, with per-chromosome read counts from all 16 chromosomes aligning well on a diagonal (Fig. 4A). Because Spo11 oligos are normalized as RPM, this result shows that the per-chromosome DSB number as a fraction of total DSBs is the same in both strains for each chromosome pair. Since there is a cell-wide homolog engagement defect in the zip3 $\Delta$ mutant (Thacker et al. 2014), all chromosomes are expected to experience increased DSBs compared with ZIP3, irrespective of homology or homeology. We therefore infer that the elimination of DSB overrepresentation on homeologous chrV was not because of reduced break formation on homeologous chrV in zip3s, but instead because the homeologous chromosomes are no longer the only ones elevated for DSB formation when all chromosomes are experiencing engagement defects.

\section{Effects of homeology and trisomy on within-chromosome DSB patterns}

Homolog engagement shapes the DSB landscape because certain subchromosomal domains respond differently to
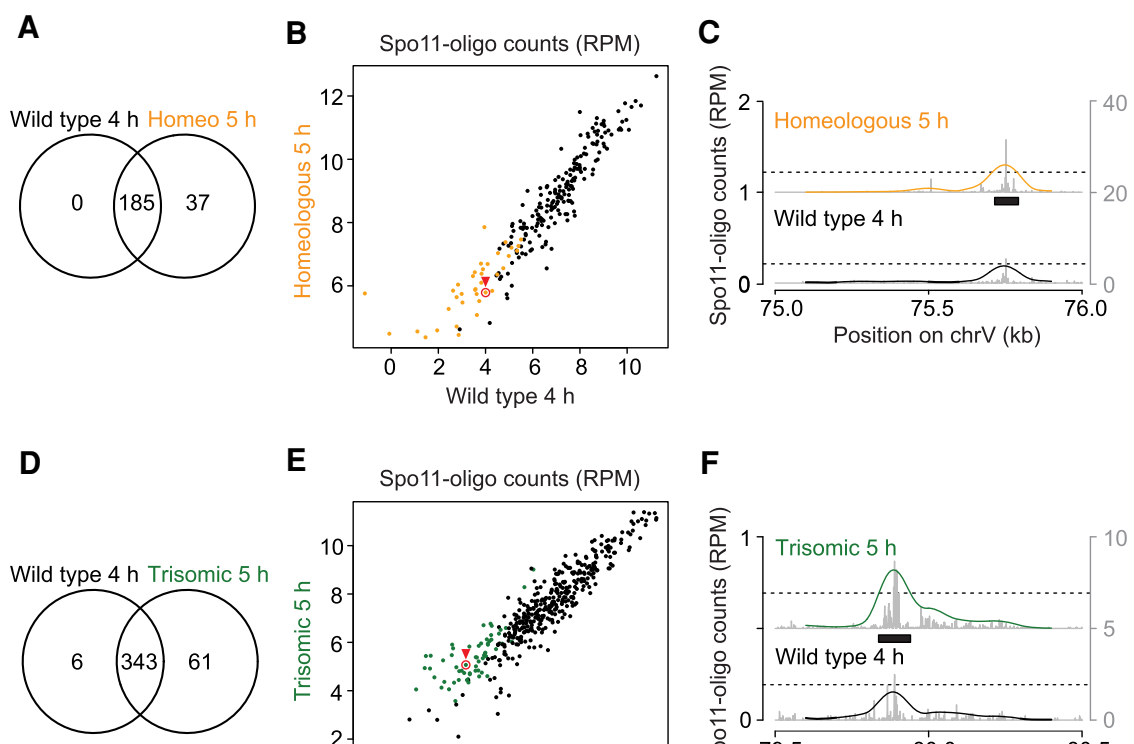

$\mathbf{E}$
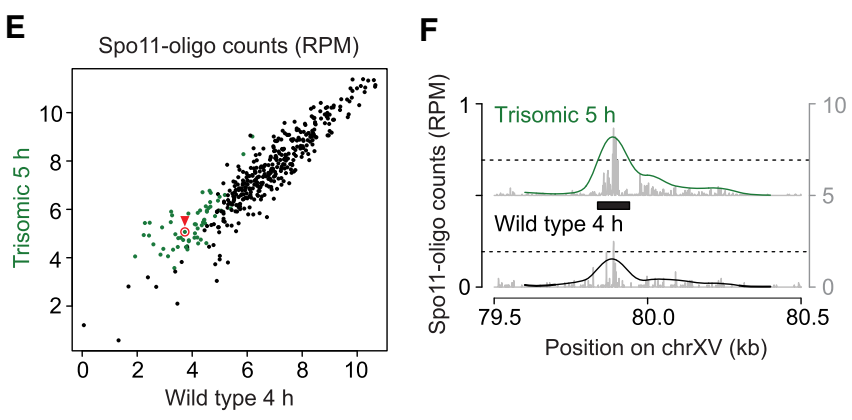

Figure 3. Conserved hot spots on homolog engagement-defective chromosomes. $(A, D)$ Venn diagrams showing the degree of overlap between hot spots called on chrV in wildtype and the homeologous strain $(A)$ or on chrXV in wild-type and the trisomy XV strain $(D)$. Hot spots were called using maps generated from time points where the total DSBs measured by Spo11-oligo labeling were maximal $(4 \mathrm{~h}$ for wild-type and $5 \mathrm{~h}$ for homeologous and trisomic strains). ( $B, C, E, F)$ Comparison of hot spot strengths. Summed Spo11-oligo read counts (nRPM, normalized to RPM after copy number correction) are shown for all hot spots called on $\operatorname{chr}(B)$ and $\operatorname{chrXV}(E)$. Orange dots are hot spots called only in the homeologous strain and green dots are those called only in the trisomic strain. The profiles for example hot spots highlighted with red circles and arrowheads are shown in $C$ and $F$. (Black bars) Boundaries of the called hot spots, (line profiles) smoothed with a 201-bp Hann window, (left $Y$-axis) nRPM of smoothed profile, (right $Y$-axis) nRPM of raw data shown in gray. 
A

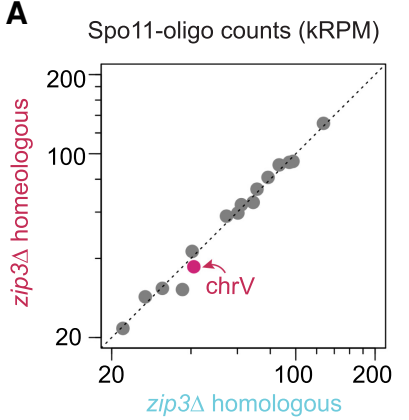

C

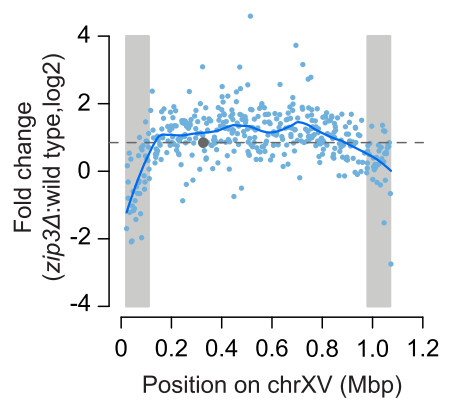

B

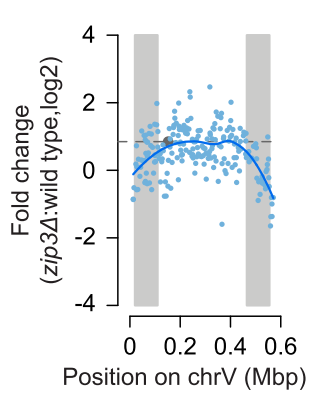

D

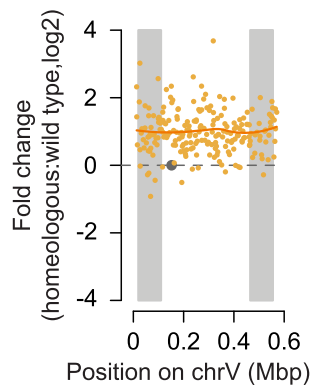

E

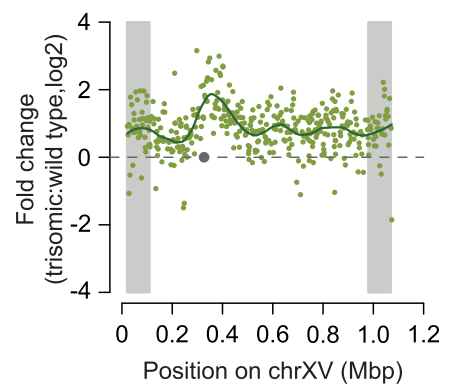

Figure 4. No DSB overrepresentation on homeologous chromosomes in a zip3 mutant background. (A) Comparison of per-chromosome Spo11-oligo totals between zip3 $\Delta$ homeologous and zip3 $\Delta$ homologous maps. $(B-E)$ Fold change of Spo11-oligo reads in hot spots along $\operatorname{chrV}(B$, $D)$ and chrXV $(C, E)$ between different strains versus wild type as labeled. For the purpose of chromosome copy number correction, the reads on homeologous chrV were doubled and the reads on trisomic chrXV were multiplied by $2 / 3$. For the zip $3 \Delta$ map a scaling factor of 1.8 -fold was applied to account for the global increase in the number of Spol1-oligo complexes in this strain (Thacker et al. 2014). Lines, local regression (loess); dashed horizontal lines, no change for $D$ or $E$, and genome average change for $B$ and $C$. (Gray circles) Centromeres, (gray shading) EARs (defined as the regions from 20 to $110 \mathrm{~kb}$ from telomeres). the zip3 $\Delta$ mutation: DSBs are increased less than the genome average in regions close to telomeres /within $\sim 20$ $\mathrm{kb}$ ), around centromeres, and flanking the ribosomal DNA (rDNA) (Thacker et al. 2014). Moreover, chromosome end-adjacent regions (EARs, from $\sim 20$ to $\sim 110 \mathrm{~kb}$ from telomeres) tend to be less sensitive to DSB suppression by homolog engagement, so on average they continue to experience DSB formation later into prophase I than is typically seen for interstitial regions (Subramanian et al. 2019).

To display regional responses to loss of feedback from homolog engagement, we plotted the ratio of Spo11-oligo counts in zip3 $\Delta$ relative to wild type within each hot spot along the lengths of chrV and chrXV (Fig. 4B,C). As expected (Subramanian et al. 2019), the EARs (shaded in gray) showed lower ratios than did interstitial parts of these chromosomes (Fig. 4B,C). In other words, because EARs are less suppressed by homolog engagement in wild type, they display less of a DSB increase in zip3 $\Delta$. Interestingly, however, we did not observe this distinct behavior for EARs when plotting ratios of homeologous chrV (Fig. 4D) or trisomic chrXV (Fig. 4E) relative to wild type. In addition, trisomic chrXV showed a disproportionately large increase in Spo11 oligos emanating from an $\sim 225-\mathrm{kb}$ region near the centromere (Fig. 4E), not seen in zip34 (Fig. 4C). Mechanisms that may account for the different responses of these chromosomes to aneuploidy as opposed to absence of Zip3 are addressed below (Discussion). Regardless of the cause of the difference, however, these results show that the specific nature of a homolog engagement defect can shape how the DSB landscape changes, in turn emphasizing the importance of reactive feedback control mechanisms in molding DSB distributions within chromosomes.

\section{Homolog engagement displaces Rec114}

To test the hypothesis that feedback from homolog engagement works through loss of DSB-promoting factors from chromosomes, we measured binding of myc-tagged Rec114 to homologous and homeologous chrV by chromatin immunoprecipitation followed by quantitative PCR (ChIP-qPCR). ChIP efficiencies (percent of input) were measured across meiotic time courses using five primer pairs targeting previously defined Rec114 ChIP peaks on $S$. cerevisiae chrV and on chrIII and chrVI as internal controls (Fig. 5A; Supplemental Fig. S4A; Supplemental Table S2; Murakami and Keeney 2014).

In wild type, Rec114 ChIP showed similar kinetics at all five loci assayed, with interpolated peak times around 3.5 h (Fig. 5B). The homeologous strain also showed similar binding profiles for the two control loci, as expected, but the three loci on homeologous chrV continued to accumulate Rec114 beyond the time when levels started to decline in wild type, reaching higher peaks at later times (Fig. 5B). As a result, the duration of Rec114 binding was lengthened (Fig. 5C; Supplemental Fig. S4B). These results support the interpretation that defects in homolog engagement result in chromosomes spending more time in a DSB-competent state.

\section{SC formation is essential for DSB suppression by homolog engagement}

Formation of both SC and crossovers is defective in ZMM mutants (Pyatnitskaya et al. 2019), so these mutants are uninformative about which of these processes is the "homolog engagement" that establishes feedback control of DSB formation. To address this question, we turned to 
A
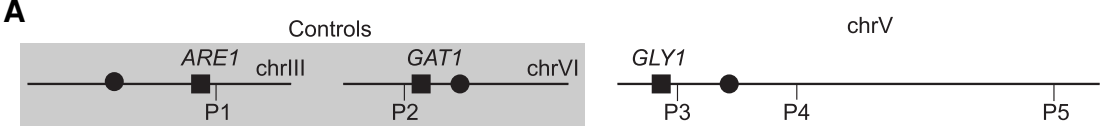

B
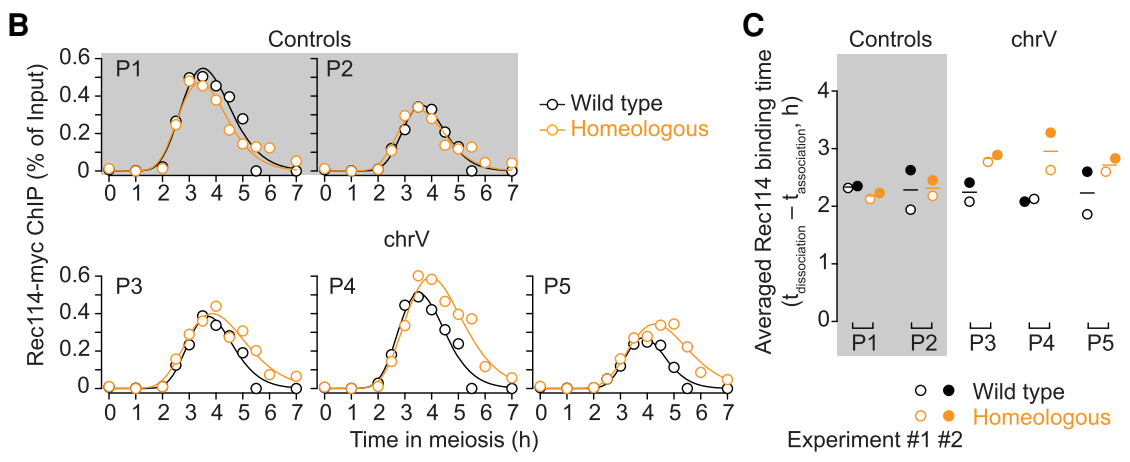

Figure 5. Persistent binding of Rec114 on homeologous chrV. (A) Primer pairs used for Rec114 ChIP-qPCR. Not to scale. P1 (on right arm of chrIII) and P2 (on left arm of chrVI) are internal controls (gray shading). P3 (left arm), P4 and P5 (right arm) are on chrV (no shading). (B) Rec114 binding kinetics for all five primer sets (experiment 1). (C) Averaged Rec114-binding duration for all five primer sets from two data sets. The binding duration is defined as dissociation time minus association time. Means are indicated by horizontal lines. Experiment 1 is shown as open circles and experiment 2 is shown as filled circles. gmc2s and ecm11 1 mutations, which separate crossing over from SC formation (Humphryes et al. 2013; VoelkelMeiman et al. 2016). Gmc2 and Ecm 11 are components of the SC central element and function as a complex in facilitating the polymerization of the transverse filament protein Zip1 (Humphryes et al. 2013). Either deletion leads to SC assembly defects, but meiotic divisions are completed efficiently with only modest delay (Supplemental Fig. S5A) and interhomolog crossovers form at elevated levels (1.1-fold to 2.8-fold higher than wild type, depending on the genetic interval assayed) (Voelkel-Meiman et al. 2016). We tested whether these mutants exhibit signatures of homolog engagement defects.

First, we examined global DSB levels by quantifying Spo11-oligo complexes. FLAG-tagged Spo11 was immunoprecipitated from meiotic cell extracts and the $3^{\prime}$ ends of Spo11 oligos were radiolabeled with terminal deoxynucleotidyl transferase and $\left[\alpha^{-}{ }^{32} \mathrm{P}\right] \mathrm{dCTP}$ before separation on SDS-PAGE (Fig. 6A,B; Supplemental Fig. S3A). Both mutants generated substantially higher levels of Spol1-oligo complexes than wild-type cultures processed in parallel, with $g m c 2 \Delta$ reaching a peak level that was 1.8 -fold higher than wild type and ecm $11 \Delta$ reaching 1.7 -fold higher (Fig. $6 \mathrm{~A}, \mathrm{~B})$. These increases are comparable with those for

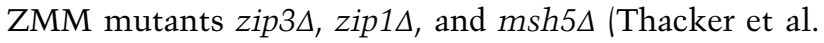
2014). The increased numbers of DSBs in ecm11 1 and gmc2 2 presumably explain much (and possibly all) of the elevated crossing over (Voelkel-Meiman et al. 2016).

Next, we compared ecm11 and zip3s Spol1-oligo maps. To evaluate similarities and differences systematically, we applied principal component analysis to perchromosome Spo11-oligo counts for multiple wild-type and mutant maps generated in this study and previous ones (Fig. 6C; Thacker et al. 2014; Zhu and Keeney 2015; Mohibullah and Keeney 2017; Murakami et al. 2020). The first and second principal components (PC1 and PC2) together accounted for $89.8 \%$ of the variance among the data sets. PC1 separated both ecm11 and zip3 $\Delta$ mutants from wild type, indicating that these mutants share DSB landscape features in common with one another. This conclusion was reinforced by hierarchical clustering, which grouped ecm11 $\Delta$ and zip3 $\Delta$ data sets with one another separately from wild type (Supplemental Fig. S5B).

Per-chromosome Spo11-oligo densities are negatively correlated with chromosome length in wild type, and this correlation collapses in a zip3 $\Delta$ mutant (Fig. 6D; Thacker et al. 2014). Loss of chromosome-size-dependent regulation of DSB numbers is thus a hallmark of defects in homolog engagement (Keeney et al. 2014; Thacker et al. 2014; Subramanian et al. 2019; Murakami et al. 2020). The ecm11 mutant showed this same hallmark (Fig. 6D). In the principal component analysis, factor loadings for PC1 correlated with chromosome size for most chromosomes (Supplemental Fig. S5C). Since PC1 was the dimension that grouped ecm $11 \Delta$ and zip3 $\Delta$ together away from wild type, we infer that loss of chromosome-size control of DSBs is a major component of the similarity between ecm11 1 and zip3 $\Delta$.

In contrast to global effects from eliminating trans-acting factors like Ecm11 or Zip3, presence of one homeologous pair should not eliminate the negative correlation of DSB density with chromosome size for the rest of the genome, because homolog engagement functions chromosome autonomously. Indeed, the homeologous strain retained the overall negative correlation except for chrV (Fig. 6D).

Spo11-oligo maps in ecm11 also displayed patterns similar to zip3 $\Delta$ within specific sub-chromosomal domains: Spo11-oligo levels were increased less than genome average in regions near telomeres, centromeres, and the rDNA array on chrXII (Fig. 6E; Supplemental Fig. S5D-F).

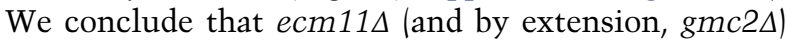
phenocopies multiple signatures of the DSB dysregulation previously documented in zip3 $\Delta$. Because ecm11 11 and gmc $2 \Delta$ mutants are SC-deficient but crossover-proficient, these results strongly indicate that $\mathrm{SC}$ formation is essential for DSB regulation by homolog engagement and that crossover formation in the absence of SC is not sufficient to down-regulate DSB formation.

\section{Discussion}

We showed here that individual yeast chromosomes unable to engage with a homologous partner continue to 

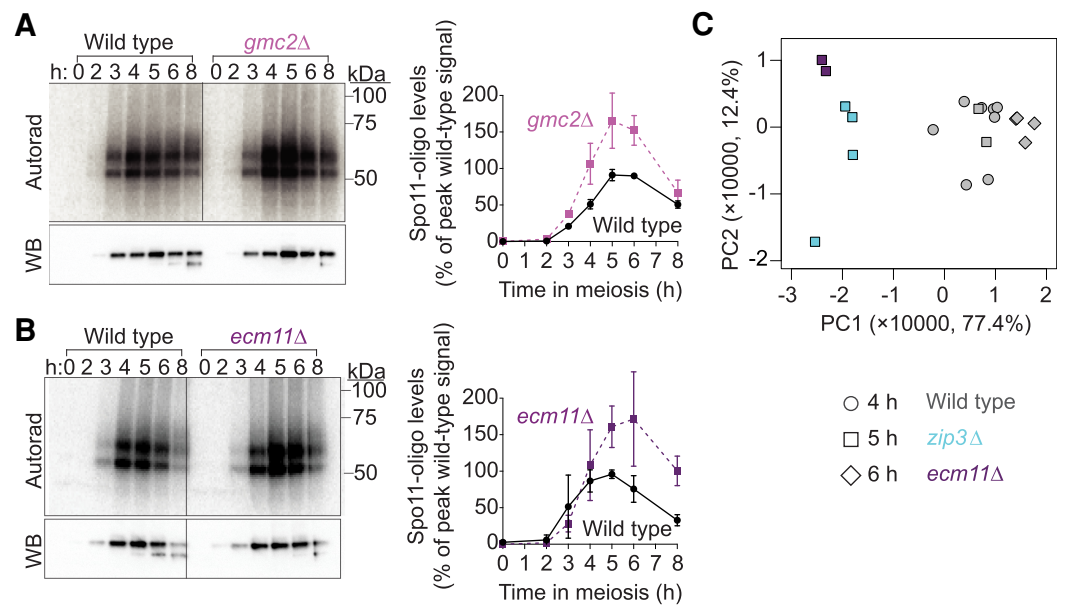

D
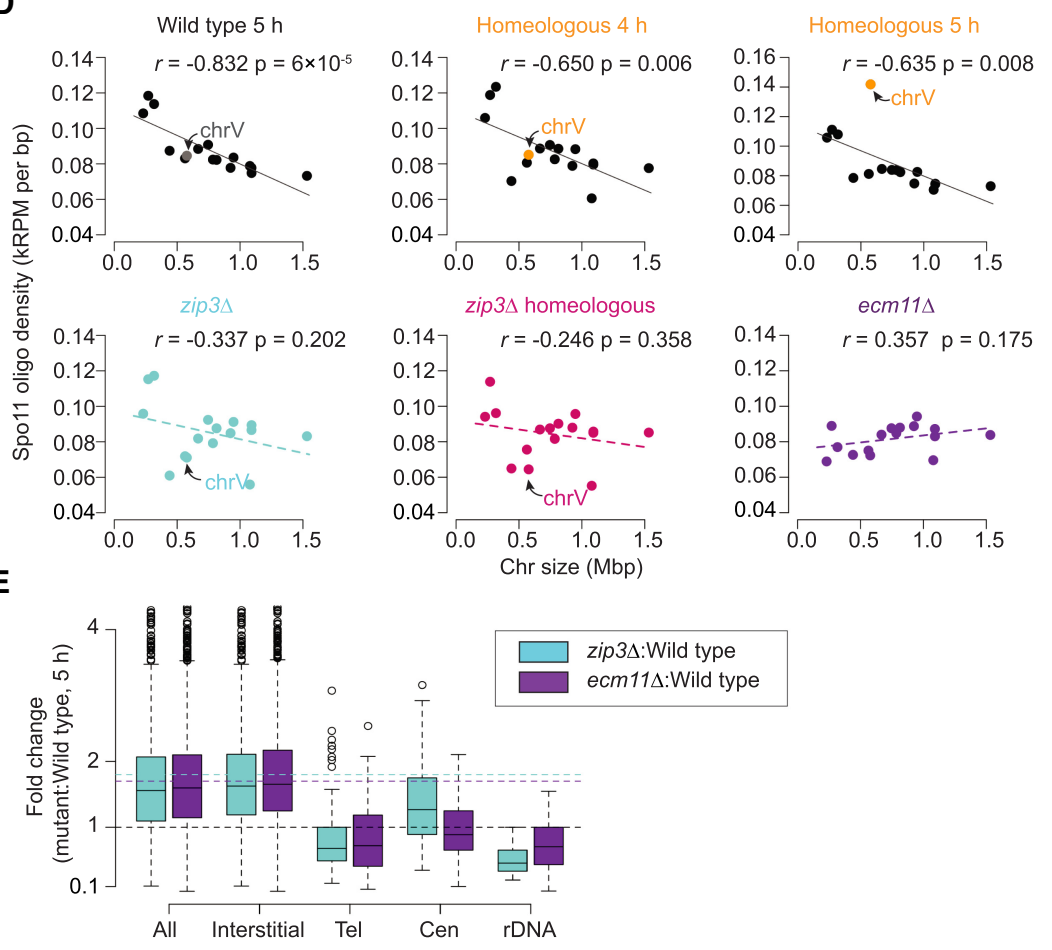

Figure 6. gmc2s and ecm $11 \Delta$ mutants share similar homolog engagement signatures as zip3 $\Delta$. $(A, B)$ Representative labeling of Spo11oligo complexes in $g m c 2 \Delta(A)$ and $e c m 11 \Delta(B)$ mutants and quantification relative to wild-type cultures processed in parallel. Error bars indicate mean \pm SD for three cultures. Radiolabeled Spo11-oligo complexes are detected by autoradiography (top, left panel) and total Spoll is detected by anti-FLAG Western blot (WB; bottom left panel). The two main labeled species differ in the sizes of oligos (Neale et al. 2005). Most Spo11 protein does not end up making DSBs, so nearly all of the visible Western blot signal is from free Spol1 that does not have an oligo attached (Neale et al. 2005). (C) Principal component analysis of 20 wild-type and mutant Spo11oligo maps. (D) Loss of anticorrelation between chromosome length and DSB density in homolog engagement-defective mutants. Each point is one chromosome. Correlation coefficients (Pearson's $r)$ are shown. (E) Fold change of Spo11-oligo counts in different chromosomal domains. Tel, within $20 \mathrm{~kb}$ of telomeres; Cen, within $10 \mathrm{~kb}$ of centromeres; rDNA, from $60 \mathrm{~kb}$ left to $30 \mathrm{~kb}$ right of rDNA; Interstitial, all others. Horizontal dashed lines mark values assumed as no change (black) and average change (1.8-fold for zip3s [cyan]; 1.7-fold for ecm11 [purple]). Boxes indicate median and interquartile range, whiskers indicate the most extreme data points that are $\leq 1.5$ times the interquartile range from the box, and individual points are outliers. accumulate DSBs past the time when other (homolog engagement-proficient) chromosomes in the same cell have largely stopped breaking. By examining crossover-proficient mutants that are unable to make mature SC because they lack key components of the central element, we also provide evidence that synapsis per se is required for homolog engagement-mediated DSB suppression. Lee et al. (2020) have provided independent and distinct evidence for increased DSB formation in gmc2 and ecm11 mutants; our results agree well with theirs. These findings support the conclusion that feedback control of DSB formation in yeast works in a chromosome-autonomous fashion as a response to SC formation, consistent with cytological evidence in mice (Wojtasz et al. 2009; Kauppi et al. 2013) but apparently distinct from nucleus-wide responses to crossover defects in C. elegans and possibly
D. melanogaster (Carlton et al. 2006; Joyce and McKim 2010; Rosu et al. 2013; Stamper et al. 2013; Crown et al. 2018).

One key difference between these species is that DSB formation precedes and is required for homologous synapsis in yeast and mice (Alani et al. 1990; Baudat et al. 2000; Romanienko and Camerini-Otero 2000), whereas DSB formation usually occurs after SC formation and is dispensable for homologous synapsis in $C$. elegans and $D$. melanogaster (Dernburg et al. 1998; McKim et al. 1998). Thus, the presence of SC can serve for cells to sense whether DSB formation has served its purpose in yeast and mice, but would not be informative for this in C. elegans and $D$. melanogaster. These findings illustrate how evolutionarily distinct strategies for completing the meiotic program can place different constraints on the 
mechanisms available for cells to ensure that DSBs form where they are needed but stop being formed where they have already done their job.

Our findings further support the interpretation that homolog engagement works by promoting the dissociation of DSB-promoting factors from chromosomes after SC formation (Wojtasz et al. 2009; Carballo et al. 2013; Keeney et al. 2014; Subramanian et al. 2019; Murakami et al. 2020). In mice, axis proteins HORMAD1 and HORMAD2 are displaced from all synapsed axes, dependent on the $\mathrm{AAA}^{+}$ATPase TRIP13 (Wojtasz et al. 2009). Synapsis also displaces specialized assemblies of SPO11-accessory proteins REC114 and MEI4 that form on the pseudoautosomal region, the only part of the $\mathrm{X}$ and $\mathrm{Y}$ chromosomes that recombines in male meiosis (Acquaviva et al. 2020). In yeast, the TRIP13 ortholog Pch2 also directs relocalization of Hop1 (ortholog of HORMAD1) after SC formation (Börner et al. 2008). Moreover, many proteins that promote Spo11 activity (Mei4, Rec102, Rec104, Rec114, and Red1) all dissociate from synapsed chromosomes (Keeney et al. 2014). A plausible scenario is that Pch2 is recruited or activated in the context of SC and disrupts the interaction between the HORMA domain in Hop 1 and its direct binding partner, the "closure motif" in Red1 (Kim et al. 2014). This locally terminates the DSB competent state by dissociating or remodeling Hopl concomitant with dissociation of DSB-promoting proteins.

While our study argues that crossing over in the absence of SC is not sufficient in yeast to provoke DSB suppression via homolog engagement, we cannot rule out the possibility that crossover formation might cooperate with SC and play some role in regulating DSB formation. Although this question has not been directly addressed in mice either, it is noteworthy that the SC that forms between nonhomologous chromosomes in the absence of recombination in a Spo11 $11^{-/-}$mutant is sufficient to provoke displacement of HORMAD1 and HORMAD2 (Wojtasz et al. 2009) and chromosome axis remodeling plus REC114 displacement from the pseudoautosomal region (Acquaviva et al. 2020). This is consistent with the possibility that synapsis is both necessary and sufficient to down-regulate DSB formation.

TRIP13-dependent displacement of HORMAD1 and HORMAD2 in mice occurs with some delay after SC formation (Wojtasz et al. 2009), implying that down-regulation of DSB formation is also not instantaneous upon completion of synapsis. This delay might simply reflect the time necessary for TRIP13/Pch2 to be recruited and to work, or it might indicate that some time-dependent change in the SC is needed as the signal for TRIP13/ Pch2 to act. In this context, we note that progression of recombination toward crossover formation is associated with pronounced changes in SC structure in C. elegans (e.g., Pattabiraman et al. 2017; Rog et al. 2017). A view that might unify DSB control systems in C. elegans, yeast, and mice might be that "maturation" of SC structure (tied to crossing over in C. elegans but perhaps tied only to the act of synapsis itself in yeast and mice) is a conserved signal for local down-regulation of DSB formation.
Different chromosomal subdomains respond differently to the defect in DSB regulation in ZMM mutants (Thacker et al. 2014). To our surprise, even though zip3 mutation was epistatic with homeology, we found that the karyotypic abnormalities we examined did not cause the same subchromosomal changes as a zip3 mutation. We envision two nonexclusive possibilities to account for the different responses of these chromosomes to aneuploidy as opposed to absence of Zip3. First, these aneuploidies may cause less severe homolog engagement defects along the entire chromosome lengths than zip3 mutation does. In this scenario, the aneuploid chromosomes retain some residual DSB suppression, so interstitial regions experience less of an increase in DSB formation compared with zip3 $\Delta$. Second, interstitial regions on the aneuploid chromosomes may retain more residual homolog engagement compared with EARs; for example, if there is still some degree of interstitial SC formation in a fraction of cells in the population. Zip1 mediates homology-independent coupling of centromeres during prophase I (Kemp et al. 2004; Newnham et al. 2010), so it is possible that the homeologous centromeres might pair and sometimes nucleate SC. Moreover, although SC is restricted to homologous pairs in normal diploids, other synaptic configurations can occur, for example, between homologous or nonhomologous chromosome segments in haploid, triploid, tetraploid, and hybrid yeast strains /Gillies et al. 1974; Moens and Ashton 1985; Loidl et al. 1991; Loidl 1995; Lorenz et al. 2002). In the majority of these cases, partial SC is produced along with unsynapsed axes and partner switches. Therefore, partial SC formed between the homeologous pair could possibly activate homolog engagement feedback to some extent.

A striking finding was the high degree of DSB overrepresentation around the centromere on trisomic chrXV. In fully triploid strains, triple-synapsed trivalents, "II + I" synapsis, and trivalents with partner switches have been observed (Loidl 1995), but the synaptic configuration of a single trisomic chromosome set is not known. We speculate that the pericentromeric region might be frequently left unsynapsed in trivalent configurations. Regardless of the cause, however, our findings suggest that trisomy antagonizes operation of the pathways that normally function to suppress DSB formation and crossing over near centromeres (Vincenten et al. 2015).

Although we focused on the increase in DSB formation that accompanies defects in homolog engagement, it is important to emphasize that engagement-defective chromosomes do not simply continue to make more and more DSBs indefinitely. Other systems also come into play to restrict the amount of DSB formation that can occur, including feedback dependent on activation of the DSB-responsive kinase Tell (ATM in mammals) and a global shutdown in DSB formation tied to exit from the pachytene stage driven by the $\mathrm{Ndt} 80$ transcription factor (Keeney et al. 2014). The complex interplay between multiple DSB regulatory pathways helps to explain how the meiotic program can be robust in the face of whole chromosome aneuploidy as examined here, and possibly in similar situations in humans such as Down syndrome 
(trisomy 21), Turner syndrome (45, XO), and Klinefelter syndrome $(47, \mathrm{XXY})$. These considerations likely also apply to challenges posed by heterozygosity for translocations or large chromosomal deletions, insertions, duplications, or inversions, and by decreased homology between chromosomes in outcrosses. Our findings thus illustrate basic principles that contribute to the fidelity of the meiotic program.

\section{Materials and methods}

Yeast strains

Yeast strains used in this study are of the SK1 background (Supplemental Table S3). SPO11 was C-terminally tagged with three FLAG epitope repeats by targeted integration of a 6 His-3FLAGlox $P$-kanMX-lox $P$ construct amplified from an S. cerevisiae SK1 SPO11-FLAG strain provided by Kunihiro Ohta (University of Tokyo) in the following strains: homeologous strain, monosomic strain, zip3 $\Delta$ mutant, zip3 $\Delta$ homeologous mutant, gmc2 $\Delta$, and ecm11 $\Delta$ mutant. In the trisomic strain, Spol1 was protein-A (PrA)-tagged (Thacker et al. 2014). We also used published Spol1 oligo maps generated using FLAG-tagged Spol1 in wild-type (Zhu and Keeney 2015; Murakami et al. 2020) and PrA-tagged Spo11 in wild-type and zip3 $\Delta$ strains (Thacker et al. 2014; Mohibullah and Keeney 2017). The nourseothricin drug-resistant marker, natMX4, was inserted near the end of the right arm of chrV (the convergent intergenic region between PUG1 and YER186c, corresponding to coordinates 560580-1 of S288C genome assembly from SGD [Saccharomyces Genome Database]) in a wild-type SK1 haploid strain. Yeasts were transformed using standard lithium acetate methods. Correct tagging was verified by PCR and Southern blot. The zip3, gmc2, and ecm11 deletion mutants were generated by replacing the coding sequences with the hygromycin B drug resistance cassette (hphMX4) individually through yeast transformation. Gene disruption was verified by Southern blot.

The homeologous strain contains one copy of $S$. pastorianus chrV in an otherwise SK1 background. S. pastorianus (lager-brewing yeast) is a hybrid of $S$. cerevisiae and $S$. eubayanus (Monerawela and Bond 2018). The copy of chrV introgressed from $S$. pastorianus into $S$. cerevisiae is originally derived from S. eubayanus. An S. cerevisiae SK1 haploid strain with chrV replaced by $S$. pastorianus chrV (marked with ilv1) was a gift from M. Lichten (Goldman and Lichten 2000). This strain was crossed with an SK1 haploid strain (chrV marked with natMX4) to create the homeologous diploid strain immediately before preparing premeiotic and meiotic cultures.

The trisomic chrXV strain arose spontaneously. A single clone obtained from the frozen stock of a wild-type strain was expanded and cultured in presporulation and sporulation media, and samples were collected for Spo11-oligo mapping and PFGE as described previously (Mohibullah and Keeney 2017) and below. The karyotype was evaluated by quantitative Southern blotting of the PFGE samples using GIT1 (chrIII) and ARG1 (chrXV) open reading frames as probes. Other independent clones obtained contemporaneously from the same frozen stock were euploid, and we later attempted unsuccessfully to isolate additional trisomic clones from this stock.

The monosomic chrV strain was generated as follows (Supplemental Fig. S1). A strong GAL1 promoter, marked with the Kluyveromyces lactis URA3 gene, was inserted adjacent to centromere DNA to create a conditional centromere (Hill and Bloom 1987). Plasmid pCEN05-UG containing a chrV centro- mere-destabilizing cassette was provided by R. Rothstein (Reid et al. 2008). The CEN5 targeting fragment was liberated by NotI digestion and transformed into an SK1 haploid to replace the native CEN5, confirmed by Southern blot. A previous study suggested that chromosome loss could be followed by endo-reduplication of the remaining chromosome, especially for small chromosomes, but chrV was not directly analyzed (Reid et al. 2008). A presporulation procedure was specifically developed to allow high frequency of chromosome loss (see "Culture Methods"), with loss of chrV and endoreduplication events closely monitored by tetrad dissection and spore clone genotyping (Supplemental Fig. S1; Supplemental Table S1).

\section{Culture methods}

Synchronous meiotic cultures were prepared using the SPS pregrowth methods as described (Murakami et al. 2009) for all strains in various culture volumes required for different experimental purposes, except for the trisomic strain using the YPA pregrowth (Alani et al. 1990) and for the monosomic chrV strain, which is slightly modified and addressed in the next paragraph. Two stages of SPS pregrowth culturing were performed to reproducibly obtain appropriate cell density before transferring into sporulation medium (SPM) for better synchrony. In brief, cells from a 4-mL saturated overnight YPD (1\% yeast extract, $2 \%$ peptone, $2 \%$ glucose) were used to inoculate into $25 \mathrm{~mL}$ of SPS (0.5\% yeast extract, $1 \%$ peptone, $0.67 \%$ yeast nitrogen base without amino acids, $1 \%$ potassium acetate, $0.05 \mathrm{M}$ potassium biphthalate at $\mathrm{pH} 5.5,0.002 \%$ antifoam 204 [Sigma]) to a density of $5 \times 10^{6}$ cells $/ \mathrm{mL}$ and cultured at $250 \mathrm{rpm}$ for $7 \mathrm{~h}$ at $30^{\circ} \mathrm{C}$. Cells were then inoculated into an appropriate larger volume of fresh SPS (200 mL for PFGE and labeling of Spo11-oligo complexes; $2 \times$ $800 \mathrm{~mL}$ for Spo11-oligo mapping) at a density of $3 \times 10^{5}$ cells/ $\mathrm{mL}$ and cultured at $250 \mathrm{rpm}$ for $12-16 \mathrm{~h}$ at $30^{\circ} \mathrm{C}$ until the density reached $3 \times 10^{7}$ to $4 \times 10^{7}$ cells $/ \mathrm{mL}$. Cells were collected by centrifugation or filtration, washed with $2 \%$ potassium acetate, then resuspended at $4 \times 10^{7}$ cells $/ \mathrm{mL}$ in an appropriate volume of SPM ( $2 \%$ potassium acetate, $0.001 \%$ polypropylene glycol; $100 \mathrm{~mL}$ for PFGE and Spo11-oligo complexes labeling; 11 for Spo11-oligo mapping) supplemented with $0.32 \%$ amino acid complementation medium $1.5 \%$ lysine, $2 \%$ histidine, $2 \%$ arginine, $1 \%$ leucine, $0.2 \%$ uracil, $1 \%$ tryptophan). Cultures were incubated in a $30^{\circ} \mathrm{C}$ shaker at $250 \mathrm{rpm}$ to induce sporulation. Samples were collected at desired times after transferring into SPM.

One round of 13.5 -h culture in YPA ( $1 \%$ yeast extract, $2 \%$ peptone, $1 \%$ potassium acetate) was used in the place of the SPS pregrowth for sporulating the trisomic strain.

The modifications for the monosomic strain were as follows: Single colonies were patched on YP-galactose plates for $24 \mathrm{~h}$ at $30^{\circ} \mathrm{C}$, then to freshly made 5-FOA plates (containing $2 \mathrm{~g} / \mathrm{L} 5$-fluoroorotic acid) for $24 \mathrm{~h}$ at $30^{\circ} \mathrm{C}$. Standard synchronous meiotic cultures were then prepared as described above. Samples were collected at $24 \mathrm{~h}$ for tetrad dissection. Complete loss of one copy of chrV should yield tetrads with 2:2 (live:dead) segregation, with all viable spores lacking the Kl-URA3 marker. Endoreduplication of chrV would give four viable ura3 spores. Failure to induce centromere loss with yield four viable spores with 2:2 segregation patterns for the K1-URA3 marker (Supplemental Fig. S1). Only cultures for which all tested tetrads had the pattern expected for complete loss and lack of endoreduplication were processed for downstream applications (Supplemental Table S1).

To check meiotic divisions, aliquots were collected at various times from synchronous meiotic culture, fixed in $50 \%(\mathrm{v} / \mathrm{v})$ ethanol, and stained with $0.05 \mu \mathrm{g} / \mathrm{mL} \mathrm{4}$, 6-diamidino-2- 
phenylindole (DAPI). Mononucleate, binucleate, and tetranucleate cells were scored by fluorescence microscopy.

\section{Detection of meiotic DSBs by Southern blot}

Genomic DNA was prepared in plugs of low-melting point agarose as described (Borde et al. 2000; Murakami et al. 2009) to avoid random shearing. The high-molecular-weight DNA was separated by PFGE as described (Borde et al. 2000; Murakami et al. 2009) and then probed by Southern blot using a radiolabeled DNA fragment within the CHA1 coding sequence located near the left arm end of chrIII (internally controlled chromosome for PFGE) or the natMX4 cassette on S. cerevisiae chrV. Signals were detected by PhosphorImager, and quantified with ImageGauge software (Fujifilm). DSB frequencies at different time points were calculated as percentages of broken molecule signals divided by paternal signals plus broken molecule signals in each lane. Observed DSB frequencies were Poisson corrected as described before (Murakami and Keeney 2014) for correction of the situation that multiple DSBs happened on each chromosome.

\section{End-labeling Spo11-oligo complexes and Spo11-oligo mapping}

Spo11-oligo complexes were extracted and detected as previously described (Supplemental Fig. S3A; Thacker et al. 2014). Briefly, Spol1-oligo complexes were immunoprecipitated from wholecell extracts by using mouse monoclonal anti-FLAG M2 antibody (Sigma). Precipitated Spol1-oligo complexes were end-labeled with $\left[\alpha-{ }^{32} \mathrm{P}\right] \mathrm{dCTP}$ in a terminal deoxynucleotidyl transferase reaction, resolved by SDS-PAGE, then transferred onto PVDF membrane and visualized by PhosphorImager. Blots were probed with mouse monoclonal anti-FLAG M2 conjugated to horseradish peroxidase (Sigma) and detected by chemiluminescent (ECL ${ }^{+}$or ECL Prime, Amersham).

For Spo11-oligo mapping, sporulation cultures of different volumes (450 mL for homeologous strain; $300 \mathrm{~mL}$ for trisomic strain; $600 \mathrm{~mL}$ for all other strains) were harvested at desired time points after transferring to sporulation media. Maps were generated for this study in strains carrying SPO11-FLAG as described (Murakami et al. 2020) except for the trisomic strain carrying SPO11PrA. Previously published wild-type maps (Thacker et al. 2014; Mohibullah and Keeney 2017) were used as controls in this study.

\section{ChIP for Rec114-Myc}

Strains expressing Rec114 with C-terminal tag of eight copies of the Myc epitope (REC114-Myc) were generated as described (Murakami and Keeney 2014). Tagged Rec114 was functional, as REC114-Myc and diploids showed normal spore viability.

Samples of $50 \mathrm{~mL}\left(2 \times 10^{9}\right.$ cells $)$ were collected at desired times after transferring to $\operatorname{SPM}(0,1,2,2.5,3,3.5,4,4.5,5,5.5,6$, and $7 \mathrm{~h})$ and cross-linked with $1 \%$ formaldehyde for $30 \mathrm{~min}$ at room temperature. Cross-linking was terminated by incubation with 131 $\mathrm{mM}$ glycine for $5 \mathrm{~min}$. Cells were washed twice with ice-cold TBS, frozen with liquid nitrogen, and stored at $-80^{\circ} \mathrm{C}$. After resuspending frozen cells using $1 \mathrm{~mL}$ of lysis buffer $(50 \mathrm{mM}$ HEPES$\mathrm{KOH}$ at $\mathrm{pH} 7.5,140 \mathrm{mM} \mathrm{NaCl}, 1 \mathrm{mM}$ EDTA, $1 \%$ Triton X-100, $0.1 \%$ Na-deoxycholate, $1 \mathrm{mM} \mathrm{PMSF}, 7 \mu \mathrm{g} / \mathrm{mL}$ aprotinin, $1 \%$ protease inhibitor cocktail [Sigma], $1 \times$ Complete protease inhibitor cocktail [Roche]) with $\sim 900 \mu \mathrm{L}$ of $0.5-\mathrm{mm}$ zirconia/silica beads (BioSpec Products) in 2-mL screw-cap Eppendorf tubes, cells were then disrupted by vigorous shaking for 1 min 12 times with an intensity of $6.5 \mathrm{M} / \mathrm{sec}$ in a FastPrep24 (MP Biomedicals) to reach a cell breakage efficiency $>99 \%(99.3 \%$ in our experiment). Another $1 \mathrm{~mL}$ of lysis buffer was added after cell disruption.
Chromatin DNA in the whole-cell extracts (WCE) was sheared by sonication with " $\mathrm{M}$ " intensity, $30 \mathrm{sec}$ on/30 sec off for $15 \mathrm{~min}$ three times in Bioruptor sonication system UCD200 (Diagenode) in 15-mL polystyrene conical tubes. Insoluble cell debris was removed by centrifugation at $21,130 \mathrm{~g}$ for $5 \mathrm{~min}$ at $4^{\circ} \mathrm{C}$. WCE was further sonicated with the same condition one more time to yield average DNA size around 350 bp (range of 100-500 bp). ChIP was performed as described (Murakami and Keeney 2014).

Locations of five primer pairs for quantitative PCR (qPCR) analysis are as followed: one locus on the right arm of chrIII (P1), one locus on the left arm of chrVI (P2, near strong DSB hot spot GAT1), and three loci on chrV (P3 to P5, P3 was near strong DSB hot spot GLY1) (Murakami and Keeney 2014) (primer sequences listed in Supplemental Table S2). qPCR was performed using the LightCycler 480 SYBR Green I Master (Roche) according to manufacturer recommendations. All measurements of ChIP and mock samples were expressed relative to the standard (dilution series of corresponding input samples).

\section{Bioinformatics analysis}

Curve fitting for DSB kinetic profile To estimate the time of DSB formation, a log-normal curve defined below was fitted to the DSB frequency (percentage of lane) plotted as a function of time (in hours): $y=a+b \times \exp \left\{-[\log (x+1)-c]^{2} / d^{2}\right\}$ where $x$ is time in hours, $y$ is DSB frequency, $a$ is the background, $b$ is the peak height, $c$ is the peak position, and $d$ is the equivalent of standard deviation. We set the background parameter $(a)$ to the DSB frequency at $0 \mathrm{~h}$, then fitted the equation to the data points by least-squares to estimate the other parameters $(b, c$, and $d)$ using the "nls" function in R.

Spo11-oligo mapping analysis Sequencing (two 50-bp paired-end reads; Illumina HiSeq 2500) was performed by the Memorial Sloan Kettering Cancer Center (MSKCC) Integrated Genomics Operation. In silico clipping of library adapters and mapping to the genome was performed by the Bioinformatics Core Facility at MSKCC using a custom pipeline as described (Pan et al. 2011; Thacker et al. 2014) with modifications. A full copy of the source code is available online at http://cbio.mskcc.org/ public/Thacker_ZMM_feedback. For strains that were pure S. cerevisiae SK1 background, Spo11-oligo reads were mapped to the sacCer2 genome assembly of type strain S288C from SGD (Saccharomyces Genome Database). For the homeologous chrV strain, the sequence of $S$. eubayanus chrV (GenBank accession no. JMCK01000005.1) (Baker et al. 2015) was added as an extra chromosome to the customized pipeline. We used only the uniquely mapping reads. Analyses were performed using R version 3.4.0 or GraphPad Prism 7.0a.

Raw and processed sequence reads for new maps generated in this study (Supplemental Table S4) are deposited in the Gene Expression Omnibus (GEO) database (https://www.ncbi.nlm.nih .gov/geo; accession no. GSE152957). This accession also contains the curated maps (unique mapping reads only, normalized to reads per million mapped) in wiggle format to allow direct visualization in appropriate genome browsers; e.g., the UCSC browser (https://genome.ucsc.edu) using genome version sacCer2. Previously published maps analyzed in this study are from GEO accession numbers GSE48299, GSE67910, GSE84696, and GSE119689 (Supplemental Table S5).

Each map was normalized to the total number of reads that mapped uniquely to a chromosome (RPM; excluding reads mapping to rDNA, mitochondrial DNA, or the 2 - $\mu$ m plasmid). The chromosome copy number was corrected for the homeologous $\mathrm{chrV}$ pair by summing up the reads from of $S$. cerevisiae chrV 
copy and $S$. pastorianus chrV or by multiplying the $S$. cerevisiae chrV read count by a factor of two, as appropriate for the analysis. We corrected for chromosome copy number for trisomic chrXV by multiplying by $2 / 3$. The purpose of this was to evaluate how much of the chrXV-specific increase in Spo11 oligos was in excess of the amount that might have been expected simply from the increase in chromosome copy number.

Since these copy number corrections affect the total normalized read number, we made the following further adjustments for analytical purposes. For the homeologous strain, we calculated the total reads without chrV for both homeologous and wildtype maps and averaged them to get a standard number. Then we adjusted the read counts for each chromosome including chrV using the standard number as total reads. We did the same separately for the trisomic strain.

For the analysis of DSB distributions within chrV in the homeologous strain (data shown in Fig. 4), we could not sum the maps for the S. cerevisiae and S. pastorianus copies of chrV, so we instead doubled the reads from $S$. cerevisiae chrV. For zip3s and ecm11 maps, a scaling factor based on quantification of Spo11-oligo complexes was applied, as described in the appropriate figure legends.

DSB hot spots were defined as clusters of Spo11 oligos meeting cutoffs for cluster size and Spo11-oligo density as previously described (Pan et al. 2011). Briefly, candidate hot spots were first identified as chromosome segments where the Spol1-oligo map smoothed with a 201-bp Hann window was >0.193 RPM per base pair, which is 2.3-fold over the genome average Spo11-oligo density. Adjacent hot spots separated by $\leq 200 \mathrm{bp}$ were merged, then candidate hot spots were filtered to remove calls that were $<25$ - bp wide and/or contained $<10$ RPM total.

Principal component analysis and hierarchical cluster analysis In total, 20 maps were included in this study: 14 wild-type maps (two for 4 h with Spo11-PrA) [Thacker et al. 2014]; two time courses collected at 4, 5, and $6 \mathrm{~h}$ with Spo11-PrA [Mohibullah and Keeney 2017]; two for $4 \mathrm{~h}$ with Spo11-FLAG (Zhu and Keeney 2015), and two time courses collected at 4 and $6 \mathrm{~h}$ with Spo11-FLAG [Murakami et al. 2020]), four zip3 $\Delta$ maps (two for $5 \mathrm{~h}$ with Spo11-FLAG in this study and two for $5 \mathrm{~h}$ with Spo11-PrA [Thacker et al. 2014]); and two ecm11 14 maps with Spo11-FLAG (this study). The total number of Spo11-oligo reads (normalized to RPM) was calculated on each. Principal component analysis on the per-chromosome totals from 20 data sets was performed using the "princomp" function in R. The first three principal components accounted for $77.4 \%, 12.4 \%$, and $2.5 \%$ of the variance across these data sets, respectively. Hierarchical clustering was performed with the "hclust" function in R using Ward's D2 method.

Curve fitting for Rec114 ChIP The curve fitting method to define association and dissociation times was as described previously (Murakami and Keeney 2014). Briefly, a modified Gaussian curve was fitted to all qPCR data points to define the Rec114 ChIP signal peak position for each primer pair. Next, this peak was used to fit a saturating exponential growth (logistic) curve to just the upward slope of the ChIP profile. We defined the $t_{\text {association }}$ as the time point where the logistic curve reached $50 \%$ of the maximum. We also estimated the dissociation time of DSB protein by fitting a logistic curve to the downward slope of the ChIP profile as $t_{\text {dissociation }}$ when the logistic curve reached $50 \%$ of the maximum.

\section{Acknowledgments}

We are grateful to Michael Lichten and Rodney Rothstein for providing strains and plasmids, Miki Shinohara and Keun Kim for discussions and sharing unpublished data, Dean Dawson and Amy MacQueen for sharing unpublished data, Agnes Viale (Memorial Sloan Kettering Cancer Center [MSKCC] Integrated Genomics Core Laboratory) for sequencing, Nicholas Socci (MSKCC Bioinformatics Core Facility) for mapping sequence reads, Stewart Shuman for gifts of T4 RNA ligase, and members of the S.K. laboratory, especially Shintaro Yamada and Devanshi Jain, for discussion and comments on the manuscript. MSKCC core facilities are supported by Cancer Center Support Grant P30 CA008748. This work was supported by National Institute of General Medical Sciences grant R35 GM118092 to S.K.

Author contributions: X.M. performed experiments. X.M. analyzed Spo11-oligo map data with contributions from H.M. N.M. generated the Spo11-oligo map of the trisomy XV strain. X.M., H.M., and S.K. designed the study and wrote the paper. H.M. and S.K. supervised the research. S.K. secured funding.

\section{References}

Acquaviva L, Boekhout M, Karasu ME, Brick K, Pratto F, Li T, van Overbeek M, Kauppi L, Camerini-Otero RD, Jasin M, et al. 2020. Ensuring meiotic DNA break formation in the mouse pseudoautosomal region. Nature 582: 426-431. doi:10.1038/ s41586-020-2327-4

Agarwal S, Roeder GS. 2000. Zip3 provides a link between recombination enzymes and synaptonemal complex proteins. Cell 102: 245-255. doi:10.1016/S0092-8674(00)00029-5

Alani E, Padmore R, Kleckner N. 1990. Analysis of wild-type and rad50 mutants of yeast suggests an intimate relationship between meiotic chromosome synapsis and recombination. Cell 61: 419-436. doi:10.1016/0092-8674(90|90524-I

Baker E, Wang B, Bellora N, Peris D, Hulfachor AB, Koshalek JA, Adams M, Libkind D, Hittinger CT. 2015. The genome sequence of Saccharomyces eubayanus and the domestication of lager-brewing yeasts. Mol Biol Evol 32: 2818-2831. doi:10 $.1093 / \mathrm{molbev} / \mathrm{msv} 168$

Baudat F, Manova K, Yuen JP, Jasin M, Keeney S. 2000. Chromosome synapsis defects and sexually dimorphic meiotic progression in mice lacking Spo11. Mol Cell 6: 989-998. doi:10 .1016/S1097-2765(00)00098-8

Borde V, Goldman AS, Lichten M. 2000. Direct coupling between meiotic DNA replication and recombination initiation. Science 290: 806-809. doi:10.1126/science.290.5492.806

Börner GV, Kleckner N, Hunter N. 2004. Crossover/noncrossover differentiation, synaptonemal complex formation, and regulatory surveillance at the leptotene/zygotene transition of meiosis. Cell 117: 29-45. doi:10.1016/S0092-8674|04)00292-2

Börner GV, Barot A, Kleckner N. 2008. Yeast Pch2 promotes domainal axis organization, timely recombination progression, and arrest of defective recombinosomes during meiosis. Proc Natl Acad Sci 105: 3327-3332. doi:10.1073/pnas .0711864105

Buhler C, Borde V, Lichten M. 2007. Mapping meiotic singlestrand DNA reveals a new landscape of DNA double-strand breaks in Saccharomyces cerevisiae. PLoS Biol 5: e324. doi:10.1371/journal.pbio.0050324

Carballo JA, Panizza S, Serrentino ME, Johnson AL, Geymonat M, Borde V, Klein F, Cha RS. 2013. Budding yeast ATM/ATR control meiotic double-strand break (DSB) levels by down-regulating Rec114, an essential component of the DSB-machinery. PLoS Genet 9: e1003545. doi:10.1371/journal.pgen.1003545

Carlton PM, Farruggio AP, Dernburg AF. 2006. A link between meiotic prophase progression and crossover control. PLoS Genet 2: e12. doi:10.1371/journal.pgen.0020012 
Cheng CH, Lo YH, Liang SS, Ti SC, Lin FM, Yeh CH, Huang HY, Wang TF. 2006. SUMO modifications control assembly of synaptonemal complex and polycomplex in meiosis of Saccharomyces cerevisiae. Genes Dev 20: 2067-2081. doi:10 $.1101 /$ gad.1430406

Cooper TJ, Wardell K, Garcia V, Neale MJ. 2014. Homeostatic regulation of meiotic DSB formation by ATM/ATR. Exp Cell Res 329: 124-131. doi:10.1016/j.yexcr.2014.07.016

Crown KN, Miller DE, Sekelsky J, Hawley RS. 2018. Local inversion heterozygosity alters recombination throughout the genome. Curr Biol 28: 2984-2990.e3. doi:10.1016/j.cub.2018 .07 .004

Dawson DS, Murray AW, Szostak JW. 1986. An alternative pathway for meiotic chromosome segregation in yeast. Science 234: 713-717. doi:10.1126/science.3535068

de Massy B, Baudat F, Nicolas A. 1994. Initiation of recombination in Saccharomyces cerevisiae haploid meiosis. Proc Nat1 Acad Sci 91: 11929-11933. doi:10.1073/pnas.91.25.11929

Dernburg AF, McDonald K, Moulder G, Barstead R, Dresser M, Villeneuve AM. 1998. Meiotic recombination in C. elegans initiates by a conserved mechanism and is dispensable for homologous chromosome synapsis. Cell 94: 387-398. doi:10 .1016/S0092-8674(00)81481-6

Gillies CB, Rasmussen SW, von Wettstein D. 1974. The synaptinemal complex in homologous and nonhomologous pairing of chromosomes. Cold Spring Harb Symp Quant Biol 38: 117122. doi:10.1101/SQB.1974.038.01.015

Goldfarb T, Lichten M. 2010. Frequent and efficient use of the sister chromatid for DNA double-strand break repair during budding yeast meiosis. PLOS Biol 8: e1000520. doi:10.1371/ journal.pbio. 1000520

Goldman AS, Lichten M. 2000. Restriction of ectopic recombination by interhomolog interactions during Saccharomyces cerevisiae meiosis. Proc Nat1 Acad Sci 97: 9537-9542. doi:10 $.1073 /$ pnas.97.17.9537

Goldstein P. 1984. Triplo-X hermaphrodite of Caenorhabditiselegans-pachytene karyotype analysis, synaptonemal complexes, and pairing mechanisms. Can I Genet Cytol 26: 1317. doi:10.1139/g84-003

Henzel JV, Nabeshima K, Schvarzstein M, Turner BE, Villeneuve AM, Hillers KJ. 2011. An asymmetric chromosome pair undergoes synaptic adjustment and crossover redistribution during Caenorhabditis elegans meiosis: implications for sex chromosome evolution. Genetics 187: 685-699. doi:10.1534/ genetics.110.124958

Hill A, Bloom K. 1987. Genetic manipulation of centromere function. Mol Cell Biol 7: 2397-2405. doi:10.1128/MCB.7.7.2397

Hochwagen A, Tham WH, Brar GA, Amon A. 2005. The FK506 binding protein Fpr3 counteracts protein phosphatase 1 to maintain meiotic recombination checkpoint activity. Cell 122: 861-873. doi:10.1016/j.cell.2005.07.010

Humphryes N, Leung WK, Argunhan B, Terentyev Y, Dvorackova M, Tsubouchi H. 2013. The Ecm11-Gmc2 complex promotes synaptonemal complex formation through assembly of transverse filaments in budding yeast. PLoS Genet 9: e1003194. doi:10.1371/journal.pgen.1003194

Joyce EF, McKim KS. 2010. Chromosome axis defects induce a checkpoint-mediated delay and interchromosomal effect on crossing over during Drosophila meiosis. PLoS Genet 6: e1001059. doi:10.1371/journal.pgen.1001059

Kauppi L, Barchi M, Lange J, Baudat F, Jasin M, Keeney S. 2013. Numerical constraints and feedback control of double-strand breaks in mouse meiosis. Genes Dev 27: 873-886. doi:10 $.1101 /$ gad.213652.113
Keeney S, Lange J, Mohibullah N. 2014. Self-organization of meiotic recombination initiation: general principles and molecular pathways. Annu Rev Genet 48: 187-214. doi:10.1146/ annurev-genet-120213-092304

Kemp B, Boumil RM, Stewart MN, Dawson DS. 2004. A role for centromere pairing in meiotic chromosome segregation. Genes Dev 18: 1946-1951. doi:10.1101/gad.1227304

Kim Y, Rosenberg SC, Kugel CL, Kostow N, Rog O, Davydov V, Su TY, Dernburg AF, Corbett KD. 2014. The chromosome axis controls meiotic events through a hierarchical assembly of HORMA domain proteins. Dev Cell 31: 487-502. doi:10 $.1016 /$ j.devcel.2014.09.013

Lao JP, Cloud V, Huang CC, Grubb J, Thacker D, Lee CY, Dresser ME, Hunter N, Bishop DK. 2013. Meiotic crossover control by concerted action of Rad51-Dmc1 in homolog template bias and robust homeostatic regulation. PLoS Genet 9: e1003978. doi:10.1371/journal.pgen.1003978

Lee M-S, Higashide MT, Choi H, Li K, Hong S, Lee K, Shinohara A, Shinohara M, Kim KP. 2020. The synaptonemal complex central region modulates crossover pathways and feedback control of meiotic double-strand break formation. bioRxiv doi:10.1101/2020.05.11.089367

Loidl J. 1995. Meiotic chromosome pairing in triploid and tetraploid Saccharomyces cerevisiae. Genetics 139: 1511-1520.

Loidl J, Nairz K, Klein F. 1991. Meiotic chromosome synapsis in a haploid yeast. Chromosoma 100: 221-228. doi:10.1007/ BF00344155

Lorenz A, Fuchs J, Trelles-Sticken E, Scherthan H, Loidl J. 2002. Spatial organisation and behaviour of the parental chromosome sets in the nuclei of Saccharomyces cerevisiae $\times$ S. paradoxus hybrids. I Cell Sci 115: 3829-3835. doi:10.1242/jcs .00066

Marston AL, Wassmann K. 2017. Multiple duties for spindle assembly checkpoint kinases in meiosis. Front Cell Dev Biol 5: 109. doi:10.3389/fcell.2017.00109

Maxfield Boumil R, Kemp B, Angelichio M, Nilsson-Tillgren T, Dawson DS. 2003. Meiotic segregation of a homeologous chromosome pair. Mol Genet Genomics 268: 750-760. doi:10.1007/s00438-002-0796-9

McKim KS, Green-Marroquin BL, Sekelsky JJ, Chin G, Steinberg C, Khodosh R, Hawley RS. 1998. Meiotic synapsis in the absence of recombination. Science 279: 876-878. doi:10.1126/ science.279.5352.876

Moens PB, Ashton ML. 1985. Synaptonemal complexes of normal and mutant yeast chromosomes (Saccharomyces cerevisiae). Chromosoma 91: 113-120. doi:10.1007/BF00294054

Mohibullah N, Keeney S. 2017. Numerical and spatial patterning of yeast meiotic DNA breaks by Tell. Genome Res 27: 278288. doi:10.1101/gr.213587.116

Monerawela C, Bond U. 2018. The hybrid genomes of Saccharomyces pastorianus: a current perspective. Yeast 35: 39-50. doi: $10.1002 /$ yea.3250

Murakami H, Keeney S. 2014. Temporospatial coordination of meiotic DNA replication and recombination via DDK recruitment to replisomes. Cell 158: 861-873. doi:10.1016/j.cell .2014.06.028

Murakami H, Borde V, Nicolas A, Keeney S. 2009. Gel electrophoresis assays for analyzing DNA double-strand breaks in Saccharomyces cerevisiae at various spatial resolutions. Methods Mol Biol 557: 117-142. doi:10.1007/978-1-59745527-5_9

Murakami H, Lam I, Huang P-C, Song J, van Overbeek M, Keeney S. 2020. Multilayered mechanisms ensure that short chromosomes recombine in meiosis. Nature 582: 124-128. doi:10 $.1038 / \mathrm{s} 41586-020-2248-2$ 
Neale MJ, Pan J, Keeney S. 2005. Endonucleolytic processing of covalent protein-linked DNA double-strand breaks. Nature 436: $1053-1057$. doi:10.1038/nature03872

Newnham L, Jordan P, Rockmill B, Roeder GS, Hoffmann E. 2010. The synaptonemal complex protein, Zip1, promotes the segregation of nonexchange chromosomes at meiosis I. Proc Natl Acad Sci 107: 781-785. doi:10.1073/pnas.0913435107

Nilssontillgren T, Gjermansen C, Holmberg S, Petersen JGL, Kiellandbrandt MC. 1986. Analysis of chromosome-V and the Ilv1 gene from Saccharomyces Carlsbergensis. Carlsberg Res Commun 51: 309-326. doi:10.1007/BF02907164

Pacheco S, Marcet-Ortega M, Lange J, Jasin M, Keeney S, Roig I. 2015. The ATM signaling cascade promotes recombinationdependent pachytene arrest in mouse spermatocytes. PLoS Genet 11: e1005017. doi:10.1371/journal.pgen.1005017

Pan J, Sasaki M, Kniewel R, Murakami H, Blitzblau HG, Tischfield SE, Zhu X, Neale MJ, Jasin M, Socci ND, et al. 2011. A hierarchical combination of factors shapes the genome-wide topography of yeast meiotic recombination initiation. Cell 144: 719-731. doi:10.1016/j.cell.2011.02.009

Panizza S, Mendoza MA, Berlinger M, Huang L, Nicolas A, Shirahige K, Klein F. 2011. Spo11-accessory proteins link double-strand break sites to the chromosome axis in early meiotic recombination. Cell 146: 372-383. doi:10.1016/j.cell 2011.07.003

Pattabiraman D, Roelens B, Woglar A, Villeneuve AM. 2017. Meiotic recombination modulates the structure and dynamics of the synaptonemal complex during C. elegans meiosis. PLoS Genet 13: e1006670. doi:10.1371/journal.pgen.1006670

Perry J, Kleckner N, Borner GV. 2005. Bioinformatic analyses implicate the collaborating meiotic crossover/chiasma proteins Zip2, Zip3, and Spo22/Zip4 in ubiquitin labeling. Proc Nat1 Acad Sci 102: 17594-17599. doi:10.1073/pnas.0508581102

Pyatnitskaya A, Borde V, De Muyt A. 2019. Crossing and zipping: molecular duties of the ZMM proteins in meiosis. Chromosoma 128: 181-198. doi:10.1007/s00412-019-00714-8

Reid RJ, Sunjevaric I, Voth WP, Ciccone S, Du W, Olsen AE, Stillman DJ, Rothstein R. 2008. Chromosome-scale genetic mapping using a set of 16 conditionally stable Saccharomyces cerevisiae chromosomes. Genetics 180: 1799-1808. doi:10 .1534/genetics.108.087999

Rog O, Kohler S, Dernburg AF. 2017. The synaptonemal complex has liquid crystalline properties and spatially regulates meiot- ic recombination factors. Elife 6: e21455. doi:10.7554/eLife .21455

Romanienko PJ, Camerini-Otero RD. 2000. The mouse Spo11 gene is required for meiotic chromosome synapsis. Mol Cell 6: 975-987. doi:10.1016/S1097-2765/00/00097-6

Rosu S, Zawadzki KA, Stamper EL, Libuda DE, Reese AL, Dernburg AF, Villeneuve AM. 2013. The C. elegans DSB-2 protein reveals a regulatory network that controls competence for meiotic DSB formation and promotes crossover assurance. PLoS Genet 9: e1003674. doi:10.1371/journal.pgen.1003674

Stamper EL, Rodenbusch SE, Rosu S, Ahringer J, Villeneuve AM, Dernburg AF. 2013. Identification of DSB-1, a protein required for initiation of meiotic recombination in Caenorhabditis elegans, illuminates a crossover assurance checkpoint. PLoS Genet 9: e1003679. doi:10.1371/journal.pgen.1003679

Subramanian VV, Zhu X, Markowitz TE, Vale-Silva LA, SanSegundo PA, Hollingsworth NM, Keeney S, Hochwagen A. 2019. Persistent DNA-break potential near telomeres increases initiation of meiotic recombination on short chromosomes. Nat Commun 10: 970. doi:10.1038/s41467-019-08875-x

Thacker D, Mohibullah N, Zhu X, Keeney S. 2014. Homologue engagement controls meiotic DNA break number and distribution. Nature 510: 241-246. doi:10.1038/nature13120

Vincenten N, Kuhl LM, Lam I, Oke A, Kerr AR, Hochwagen A, Fung J, Keeney S, Vader G, Marston AL. 2015. The kinetochore prevents centromere-proximal crossover recombination during meiosis. Elife 4: e10850. doi:10.7554/eLife.10850

Voelkel-Meiman K, Cheng SY, Morehouse SJ, MacQueen AJ. 2016. Synaptonemal complex proteins of budding yeast define reciprocal roles in MutS $\gamma$-mediated crossover formation. Genetics 203: 1091-1103. doi:10.1534/genetics.115.182923

Wojtasz L, Daniel K, Roig I, Bolcun-Filas E, Xu H, Boonsanay V, Eckmann CR, Cooke HJ, Jasin M, Keeney S, et al. 2009. Mouse HORMAD1 and HORMAD2, two conserved meiotic chromosomal proteins, are depleted from synapsed chromosome axes with the help of TRIP13 AAA-ATPase. PLoS Genet 5: e1000702. doi:10.1371/journal.pgen.1000702

Zhu X, Keeney S. 2015. High-resolution global analysis of the influences of Bas1 and Ino4 transcription factors on meiotic DNA break distributions in Saccharomyces cerevisiae. Genetics 201: 525-542. doi:10.1534/genetics.115.178293 


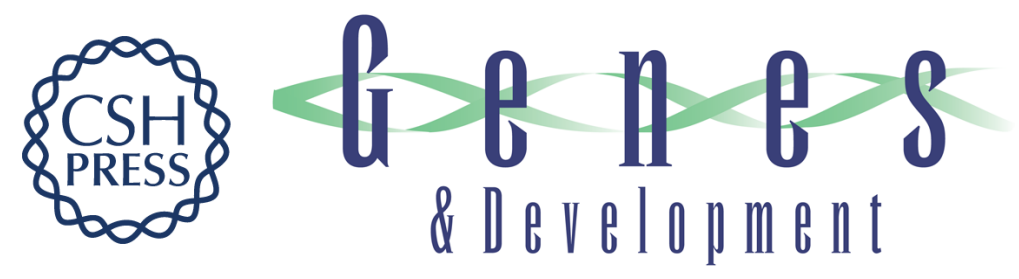

\section{Chromosome-autonomous feedback down-regulates meiotic DNA break competence upon synaptonemal complex formation}

Xiaojing Mu, Hajime Murakami, Neeman Mohibullah, et al.

Genes Dev. 2020, 34: originally published online November 12, 2020

Access the most recent version at doi:10.1101/gad.342873.120

\section{Supplemental http://genesdev.cshlp.org/content/suppl/2020/11/11/gad.342873.120.DC1 \\ Material}

Related Content

A new role for the synaptonemal complex in the regulation of meiotic recombination

Nancy M. Hollingsworth

Genes Dev. December , 2020 34: 1562-1564

References This article cites 64 articles, 21 of which can be accessed free at: http://genesdev.cshlp.org/content/34/23-24/1605.full.html\#ref-list-1

Articles cited in: http://genesdev.cshlp.org/content/34/23-24/1605.full.html\#related-urls

Creative This article, published in Genes \& Development, is available under a Creative Commons Commons License (Attribution 4.0 International), as described at License http://creativecommons.org/licenses/by/4.0/.

Email Alerting Receive free email alerts when new articles cite this article - sign up in the box at the top Service right corner of the article or click here.

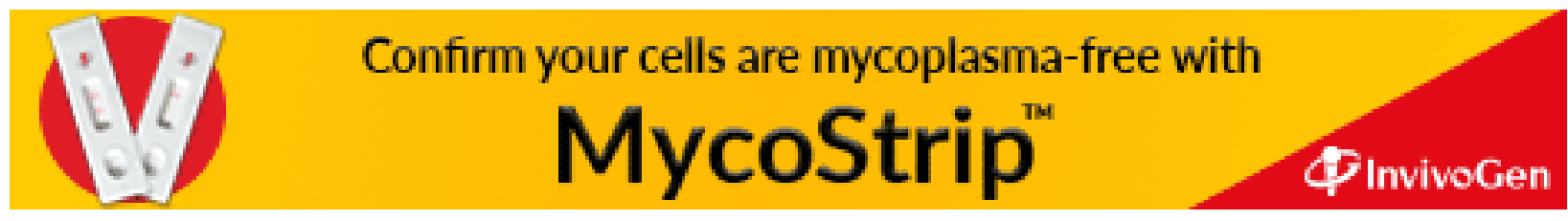

